\title{
Meet the Needs of Reservoir Identification Research of Atypical Archie Phenomenon
}

\author{
Wenqiang Tang ${ }^{1,2}$, Kun Xiong ${ }^{2}$, Chen Pan $^{2}$, Haoting Xing ${ }^{3}$, Wei Chen ${ }^{4}$, Xuan Liu ${ }^{2}$, Xinxin Zang, \\ Yun Yang ${ }^{1,6}$ \\ ${ }^{1}$ Institute of Sedimentary Geology, Chengdu University of Technology, Chengdu, China \\ ${ }^{2}$ Oil Production Plant I, Qinghai Oilfield Branch of Petrochina, Dunhuang, China \\ ${ }^{3}$ Institute of Exploration and Development, Qinghai Oilfield Branch of Petrochina, Dunhuang, China \\ ${ }^{4}$ Oil Production Plant III, Qinghai Oilfield Branch of Petrochina, Dunhuang, China \\ ${ }^{5}$ Oil Production Plant II, Qinghai Oilfield Branch of Petrochina, Dunhuang, China \\ ${ }^{6}$ Testing Company, Qinghai Oilfield Branch of Petrochina, Dunhuang, China
}

\section{Email address:}

tangwqcdut@sina.com (Wenqiang Tang),446896969@qq.com (Kun Xiong),641859727@qq.com (Chen Pan), 1454174491@qq.com (Haoting Xing),342808528@qq.com (Wei Chen),373012865@qq.com (Xuan Liu),

250355026@qq.com (Xinxin Zang),215493025@qq.com (Yun Yang)

\section{To cite this article:}

Wenqiang Tang, Kun Xiong, Chen Pan, Haoting Xing, Wei Chen, Xuan Liu, Xinxin Zang, Yun Yang. Meet the Needs of Reservoir Identification Research of Atypical Archie Phenomenon. Science Discovery. Vol. 7, No. 2, 2019, pp. 87-91. doi: 10.11648/j.sd.20190702.16

Received: April 2, 2019; Accepted: May 7, 2019; Published: May 23, 2019

\begin{abstract}
According to the core data, the characteristics of resistivity variation under different porous structure and wettability are studied and explained theoretically. It is concluded that with the increase of porous structure exponent $m$, the resistivity increases significantly with the same saturation, and the influence is beyond imagination. With the increase of saturation index $n$, the resistivity increases with the same saturation. If all fixed $m$ and $n$ values are used to calculate the water saturation of the reservoir, the water saturation result will be very high and the hydrocarbon reservoir will be ignored. This change feature is of great guiding significance to the identification of hidden oil and gas reservoir satisfying the atypical Archie formula.
\end{abstract}

Keywords: Atypical Archie Phenomenon, Porous Structure Exponent, Saturation Index, Wettability, Archie Formula

\section{满足非典型阿尔奇现象油层识别研究}

\author{
唐闻强 ${ }^{1,2}$, 熊坤 ${ }^{2}$, 潘晨 $^{2}$, 邢浩婷 $^{3}$, 陈伟 ${ }^{4}$, 刘远 $^{2}$, 㶓欣欣 ${ }^{5}$, 杨芸 ${ }^{1,6}$ \\ 1成都理工大学沉积地质研究院, 成都, 中国 \\ ${ }^{2}$ 中国石油青海油田分公司采油一厂, 敦煌, 中国 \\ ${ }^{3}$ 中国石油青海油田分公司勘探开发研究院, 敦煌, 中国 \\ ${ }^{4}$ 中国石油青海油田分公司采油三厂, 敦煌, 中国 \\ ${ }^{5}$ 中国石油青海油田分公司采油二厂, 敦煌, 中国 \\ ${ }^{6}$ 中国石油青海油田分公司测试公司, 敦煌, 中国
}

\section{邮箱}

tangwqcdut@sina.com（唐闻强），446896969@qq.com（熊坤）,641859727@qq.com（潘晨），1454174491@qq.com（邢浩婷）， 342808528@qq.com（陈伟），373012865@qq.com（刘远）, 250355026@qq.com（藏欣欣）, 215493025@qq.com（杨芸）

摘要：根据岩心资料, 研究了不同孔隙结构和润湿性下电阻率的变化特征, 并从理论上加以说明。可以得出结论, 随 着孔隙结构指数 $m$ 的增大, 相同饱和度下电阻率大幅增加, 并且影响是出乎意料的; 随着饱和度指数 $n$ 的增加, 相同饱 
和度下电阻率亦增加。如果全部用固定的 $m$ 和 $n$ 值来计算储层含水饱和度, 含水饱和度结果将非常高, 油气层将被忽略。 该变化特征对指导满足非典型阿尔奇公式的隐蔽油气层的识别具有非常大的指导意义。

关键词：非典型阿尔奇现象，孔隙结构指数，饱和度指数，润湿性，阿尔奇公式

\section{1. 引言}

在砂岩储层中, 利用测井资料计算含油饱和度的基础 是阿尔奇公式。但当储层岩性变化较为复杂时，例如在泥 质砂岩储层、复杂孔隙储层中，阿尔奇公式的使用效果则 会变差[1-3]。在松辽盆地南部, 北部湾盆地, 川西盆地等 致密储集层中, 由于孔隙结构复杂, 测井曲线有大量“非 典型Archie（阿尔奇）”现象[4-7]。

含水饱和度是评价储层含油性的重要指标, 是测井定 量解释中必不可少的参数之一, 低阻油层的评价也不例外 [8-9]。和纯净砂岩相比, 由于复杂砂岩岩性的多样化, 造 成孔隙结构、润湿性大多区别于纯净砂岩, 其岩性、电性、 含油性关系也呈现“非典型阿尔奇化”现象 [10-11]。因此, 研究区域的含水饱和度评价应基于阿尔奇方程, 通过改良、 细化、强化影响电阻率变化的主导因素 (例如采用变化的 孔隙结构指数 $m$ 、含水饱和度指数 $n$ 等方法), 建立适合研 究区地质特点的含水饱和度模型[12-14]。

储层“非典型阿尔奇化”现象就是，储层导电整体上服 从阿尔奇方程, 但存在两个非典型现象。地层因素 $F$ 和孔 隙度 $\Phi$ 在双对数坐标下呈现非线性关系; 含水饱和度 $S_{\mathrm{w}}$ 和 电阻率增大倍数I在双对数坐标下呈现多样性变化特征, 并且两者对电阻率的影响也不尽相同。

本文以某油田实际岩芯数据为例计算了孔隙结构指 数和饱和度指数对电阻率的影响, 用变化的 $m 、 n$ 值确定非 典型阿尔奇化的油层, 并通过试油验证, 为该区增储、增 产作出了贡献。

\section{2. 孔隙结构指数 $m$ 对电阻率的影响}

孔隙度结构指数 $m$ 值的分布与变化受各种因素的共 同作用, 但它们主要受岩石孔隙结构控制, 其物理意义在 于表征孔隙结构对岩石导电性的影响[15]。通过对表征岩 石孔隙结构的宏观特性分析, 确定了复杂砂岩储层 $m$ 值的 分布和变化, 主要是孔隙度与渗透率, 因此 $m$ 可表达为地 层孔隙度和渗透率的函数。对于孔隙砂岩储层, 从描述岩 石孔隙结构的微观特性分析, 则主要取决于储层孔腔与孔 喉截面积的耦合关系, 即与孔喉比的大小直接相关[16]。

\section{1. 孔隙结构指数 $m$ 的分布规律}

下图为某研究区岩电实验地层因素与孔隙度图版, 发 现研究区泥质砂岩在不同孔隙结构储层下, 具有不同的孔 隙度结构指数 $m$ 值分布 (图1), 这与纯净砂岩 $m=2$ 差别较 大。其中I类储层, $a=1$ 时, $m=1.7$; II类储层, $a=1$ 时, $m=1.44$ 。 从中可以看出, 孔隙结构的差异, 对于砂岩电阻率不同的 影响。

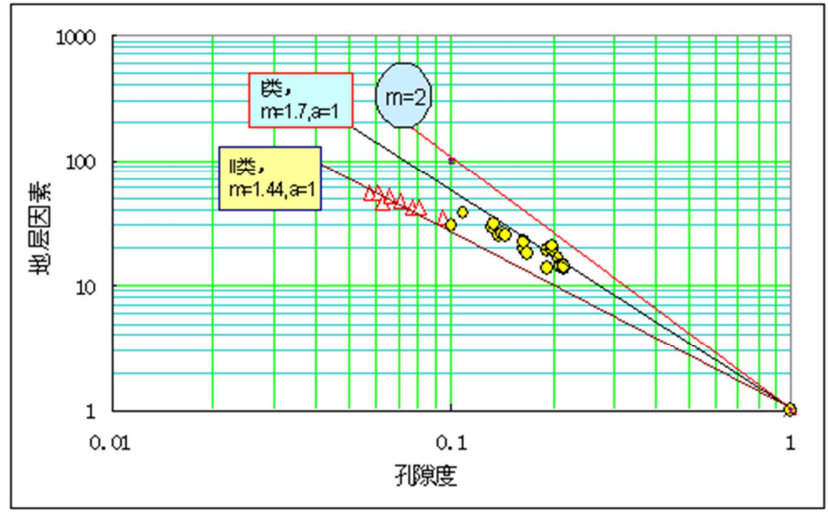

图1 不同储层分类的孔隙度与地层因素关系。

\section{2. 孔隙结构指数 $m$ 对电阻率的影响分析}

由阿尔奇公式:

$$
S_{w}=\sqrt[n]{\frac{a b R_{w}}{R_{t} \phi^{m}}}
$$

得到:

$$
R_{t}=\frac{a^{*} b^{*} R_{w}}{S_{w}{ }^{n} * \Phi^{m}}
$$

对 $m$ 求偏导：

$$
\frac{\partial R_{t}}{\partial m}=-m * \frac{a * b * R_{w}}{S_{w}^{n} * \Phi^{m+1}}
$$

式1到式3中: $S_{\mathrm{w}}$ 为含水饱和度; $a 、 b 、 m 、 n$ 为阿尔奇 公式中系数; $R_{\mathrm{w}}$ 为地层水电阻率; $R_{\mathrm{t}}$ 为地层电阻率; $\Phi$ 为 孔隙度。

假设 $a=1, b=1, n=1.6, S_{\mathrm{w}}=50 \%, R_{\mathrm{w}}=0.23$ 条件下, 得 出不同孔隙度、不同 $m$ 值对 $R_{\mathrm{t}}$ 的影响（图2）：在低孔隙度 条件下, 孔隙结构指数对电阻率的影响更大, 并且研究区 块储层孔隙度就在 $8 \%-13 \%$ 之间, 孔隙结构指数的变化对 电阻率的影响值超过 $50 \%$ 。 


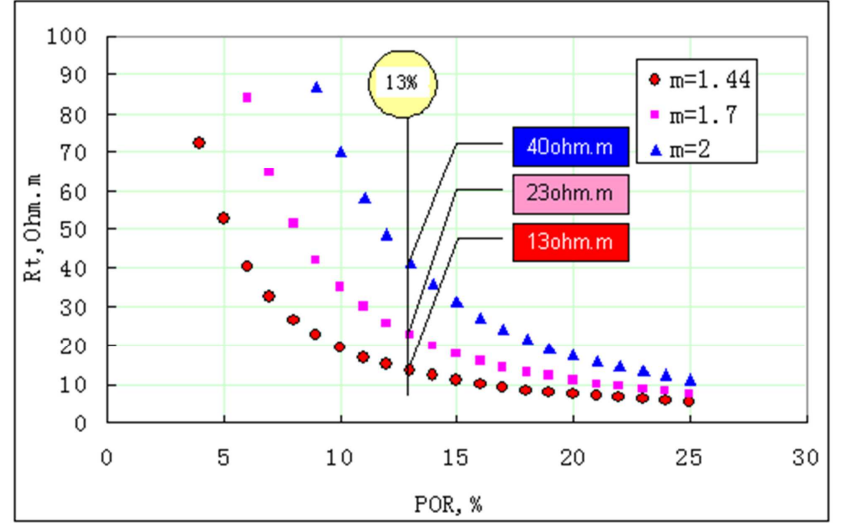

图2 不同 $m$ 下地层电阻率随孔隙度的变化关系。

从图 2 亦可知, 在其他条件相同, 孔隙度为 $13 \%$ 的条 件下, 当 $m=1.44$ 时, $R_{t}=13 \Omega \cdot m$; 当 $m=1$.7时, $R_{t}=23 \Omega \cdot m$; 当 $m=2$ 时, $R_{t}=40 \Omega \cdot m$ 。比较纯净砂岩, $\mathrm{I}$ 类储层电阻率下 降 $43 \%$, II类储层电阻率下降高达 $67.5 \%$ 。如果以研究区深 层系I类油层做为常规电阻率油层, 则相同条件下, II类油 层电阻率下降 $43.5 \%$, 接近降低一半。因此, 低孔隙结构 指数 $m$ 易形成低阻油层，该区块大部分低阻油层是由低 $m$ 值引起。

在孔隙度大的地方, $m$ 值变化对电阻率影响不显著。 在孔隙度小的地方, 孔隙结构指数 $m$ 对油层电阻率的影响 之大是显而易见的。

\section{3. 饱和度指数 $n$ 对电阻率的影响}

饱和度指数 $n$ 值的物理意义是油气饱和度微观分布状 态指数, 与岩石的润湿性息息相关。

\section{1. 润湿性和饱和度指数 $n$ 的关系}

饱和度指数 $n$ 的变化主要反映在岩石润湿性的差异上 [17]。润湿性的差异导致岩石储集空间中的油（气）微观 分布的差异, 并引起岩石导电路径和导电性的变化。根据 国内外近年来的研究资料统计, 亲水岩石的 $n$ 值一般在 1.5-2.3范围内, 亲油岩石的 $n$ 值则分布在 2.4-10之间, 甚至 更高, 表现为亲油性地层的 $n$ 值大于亲水性地层 $[18]$ 。

\section{2. 研究区储层饱和度指数 $n$ 的分布特点}

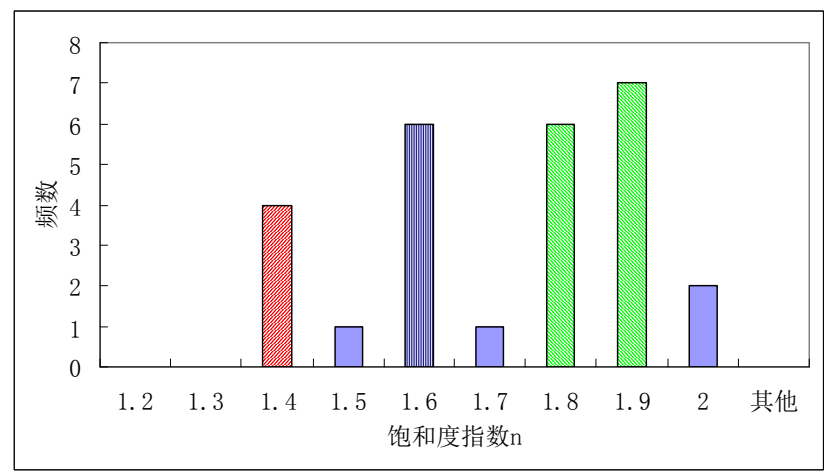

图3 岩心 $n$ 值分布图。
图3是该油田岩芯的岩电实验结果中饱和度指数统计 图。实验结果表明, 该区岩石饱和度指数 $n$ 值分布较宽, 主要为 $1.4 、 1.6 、 1.85$, 和纯净砂岩 $n$ 值为 2 差别较大。其 差异一方面体现了该地区岩石的亲水和强亲水特征, 另外 一方面也体现了岩石微观孔隙中油气分布状态的多样性。

\section{3. 饱和度指数 $n$ 对电阻率影响敏感程度分析}

面对饱和度指数 $n$ 的多样性（图3）, 充分了解 $n$ 值对 电阻率增大倍数 $I$ 的影响, 将有助于我们在实际资料处理 过程中, 把握参数的选取, 正确判别流体性质。

由阿尔奇公式（式1），对 $n$ 求偏导:

$$
\frac{\partial R_{t}}{\partial n}=-n * \frac{a * b * R_{w}}{S_{w}^{n+1} * \Phi^{m}}
$$

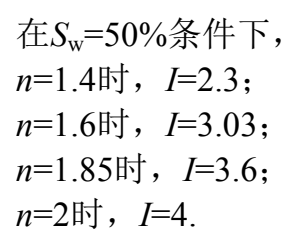

当 $a=1, b=1, m=1.7$, 含水饱和度 $S_{\mathrm{w}}=50 \%$, 得出随孔 隙度的变化, 不同 $n$ 值对 $R_{t}$ 的影响（图4）：在岩石润湿性 为亲水条件下, 更易形成低阻, 且地层越亲水, 电阻率越 低。 $n$ 值的变化不论是在中孔或者低孔的情况下, 对电阻 率的影响几乎相同, 这是由于在表面积相同的情况下吸附 在岩石上的束缚水含量一样。

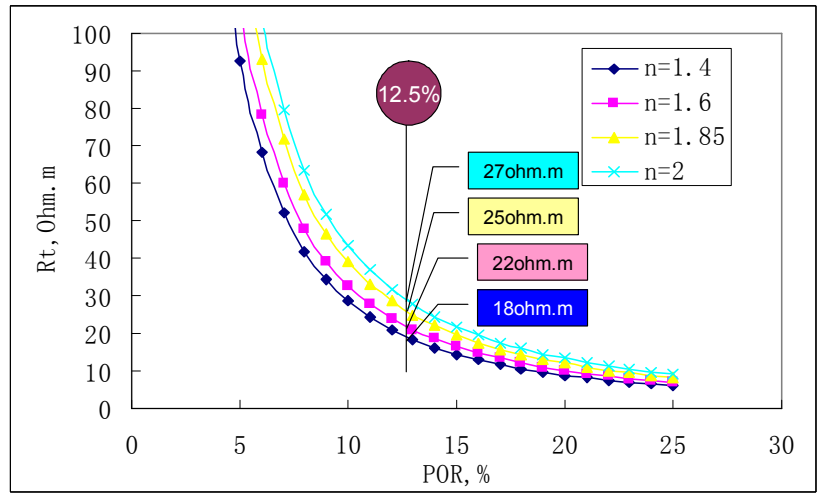

图4 不同 $n$ 下地层电阻率随孔隙度的变化关系。

从图 4 也可以看出 $n$ 值的变化对 $R_{\mathrm{t}}$ 的影响。孔隙度 $12.5 \%$ 条件下, 当 $n=1.4$ 时, $R t=18 \Omega \cdot m$; 当 $n=1$.6时, $R t=22$ $\Omega \cdot m$; 当 $n=1.85$ 时, $R t=25 \Omega \cdot m$; 当 $n=2$ 时,$R t=27 \Omega \cdot m$ 。 若以饱和度指数 $n=1.85$ 为标准, $n=1.6$ 时, 电阻率下降 $12 \% ; n=1.4$ 时, 电阻率下降 $28 \%$ 。可以看出饱和度指数 $n$ 对油层电阻率具有较大的影响。知道这种影响规律后, 在实际处理中选择参数的时候要特别注意。

\section{4. 应用实例}

孔隙度结构指数 $m$ 值受岩石孔隙结构影响, 中值半径 越大, 对应 $m$ 值增大 [19]。对于孔隙度结构指数 $m$ 值变化的 岩性成因, 主要和岩石填隙物含量密切相关。研究区的填 
隙物类型主要为方解石、粘土、和次生加大的石英。填隙 物含量的增加导致粒间孔隙的填充变得复杂, 并且薄膜滞 水和毛管滞水体积增大。因此, $m$ 值随着填隙物含量的增 加而降低。填充物越高, 越容易在孔吼中形成微细毛管发 育的优良导电通道, 导致整体电阻率下降。

体现在测井响应上, 主要表现为密度值的增大, 这也 是研究区低阻油层的特殊性所在。对测井资料进行精细分 析研究后发现, 该类储层属于II类储层, 在研究区比较普 遍的存在。图 5 为某井1863-1874.3m处, 密度值高达 $2.4 \mathrm{~g} / \mathrm{cm}^{3}$ 以上, 相对其他油层较高, 原解释结论对此说不 清楚, 目前通过分析后决定用 $m=1.44$ 计算含油饱和度, 计
算 $S_{0}=67 \%$, 建议对此试油, 实施后日产油 $2.7 \mathrm{~m}^{3}$, 不产水。 通过对全区测井资料二次解释, 发现大量此类油层。

一般说来, 饱和度指数 $n$ 与粒度中值呈正相关的关系, 粒度中值越小, 说明岩性颗粒细, 亲水性强, 使导电趋势 增强。因此 $n$ 值变化的主要电性响应特征是, 岩性变细时, 自然伽马曲线值升高或者补偿中子测井值升高。图6为研 究区某井2130-2133m层内孔隙度一致情况下, 中子升高岩 性变细, $n$ 值变小, 导致油层低阻的典型例子。我们选择 $n=1.4$ 进行计算, 该层含油饱和度为 $60 \%$, 建议试油, 试油 结论日产油 $1 \mathrm{~m}^{3}$, 不产水, 结论为差油层。

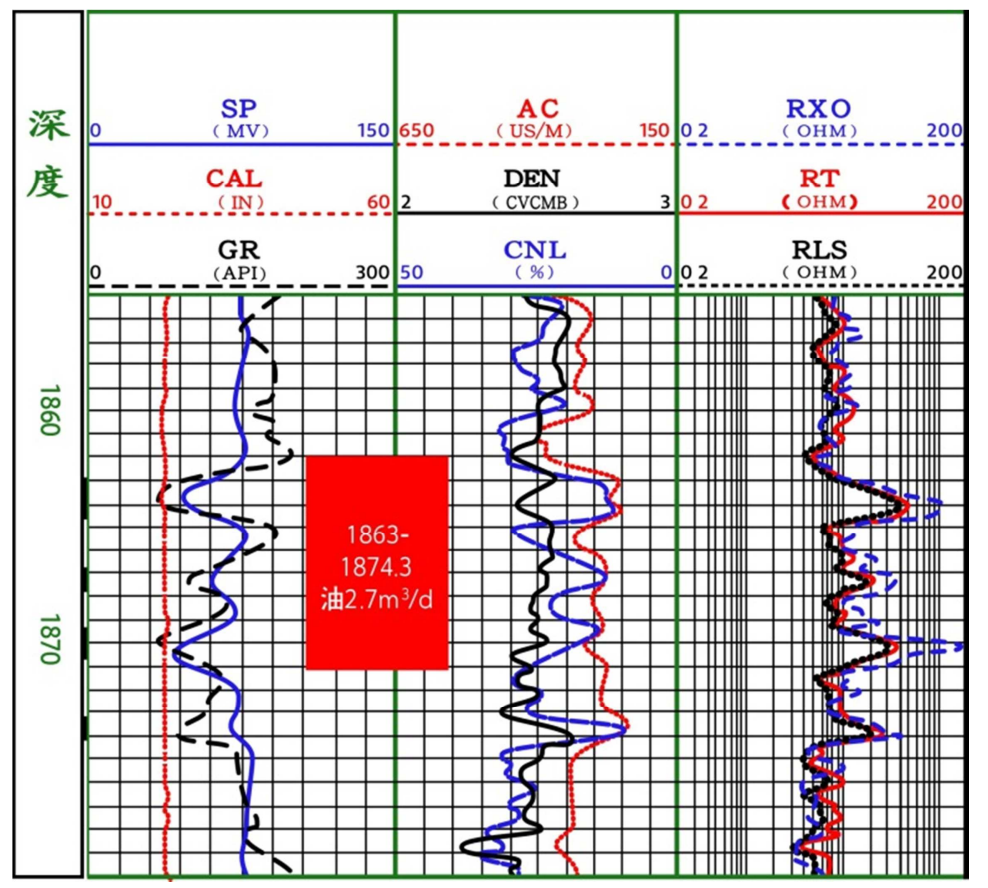

图5 某井试油油层高密度储层测井响应特征。

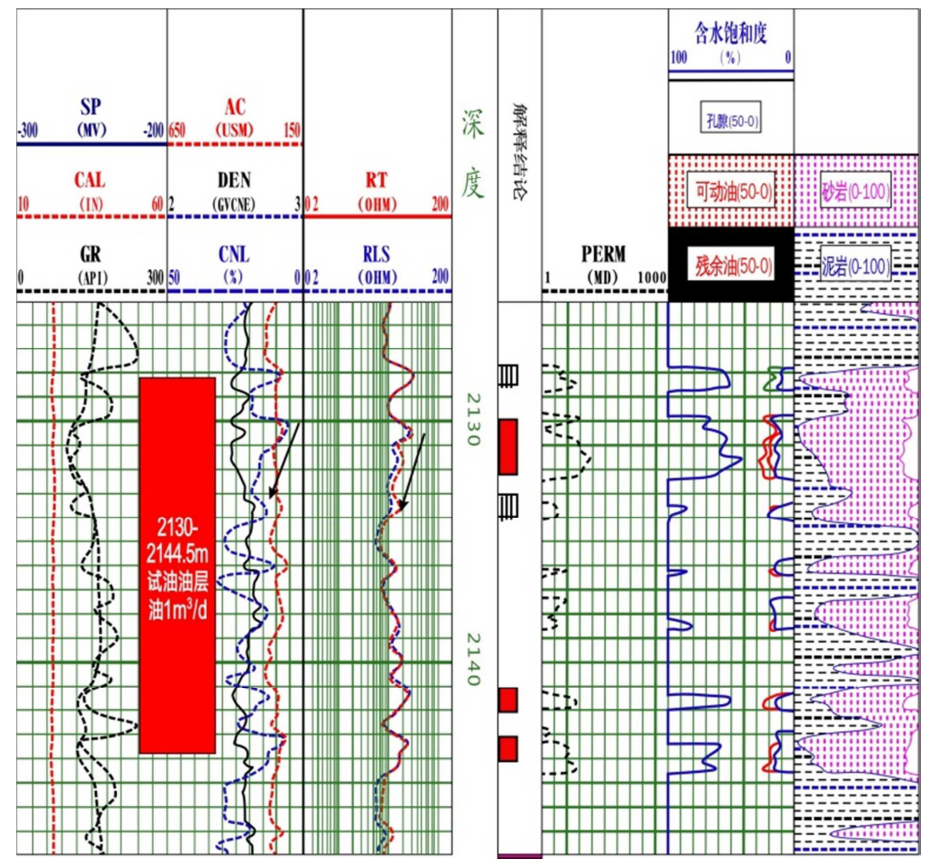

图6 某井试油油层 $n$ 值导致低阻电性响应特征。 


\section{5. 结论}

岩石电性资料在测井解释、储层评价以及储量预测中 具有重要作用, 目前, 在电法测井解释中广泛使用的仍是 阿尔奇提出的经验公式, 然而, 越来越多的实验和研究表 明简单的阿尔奇关系并不能描述所有的电性特征。如果思 路不转变, 将错失很多类似的油层。

基于变化的 $m 、 n$ 值, 用阿尔奇公式进行计算含水饱和 度, 对研究区块的老井进行普查, 发现了大量的满足非典 型阿尔奇现象的油层, 对该区的增储, 增产提供新思路, 实际应用效果非常好。

\section{基金项目}

中国石油天然气股份有限公司重大科技专项“柴达木 盆地建设高原大油气田勘探开发关键技术研究与应用” (2016E-0104GF)。

\section{参考文献}

[1] 张洁,罗健,夏瑜, 等.阿尔奇公式的适用性分析及其拓展 $[\mathrm{J}]$. 地球物理学进展,2018,61(1):311-322.

[2] 杨克兵,王竞飞,马凤芹,等.阿尔奇公式的适用条件分析及对 策[J].天然气石油,2018,36(2):58-63.

[3] 孙游雪, 高齐明,魏玉梅, 等.水淹层评价中 $R_{w} 、 m 、 n$ 值的确定 [J].山东理工大学学报 (自然科学版) ,2018,32(3):57-60.

[4] 胡胜福,周灿灿, 李霞, 等.复杂孔隙结构致密砂岩含油饱和度 梯形孔隙模型[J].石油勘探与开发,2017,44(5):827-835.

[5] 田秘,侯正猛,李占东,等.变形后阿尔奇公式在特低渗储层解 释中的应用 $[\mathrm{J}]$. 重庆理工大学学报（自然科学 版）,2017,31(10):128-133.

[6] 张恒荣, 何胜林, 郑香伟, 等. 复杂孔隙结构低阻油层含水饱和 度解释新方法 [J]. 西南石油大学学报 (自然科学 版）,2018,40(1):97-103.
[7] 司马立强,王超, 吴丰, 等. 川西马井气田蓬莱镇组致密砂岩储 层可动水饱和度计算方法[J].测井技术,2017,41(2):199-204.

[8] 孙建孟,王克文,李伟.测井饱和度解释模型发展及分析 [J].石 油勘探与开发,2008,35(1):101-107.

[9] 刘柏林, 王友启. 低含油饱和度油藏开发特征 [J]. 石油勘探与 开发,2011,38(3):341-344.

[10] 王克文,孙建孟,关继腾.油气层润湿特性对电阻率的影响规 律研究[J].天然气工业,2006,26(12):86-88.

[11] Wang Kewen, Sun Jianmeng, Guan Jiteng, et al. Percolation Network Modeling of Electrical Properties of Reservoir Rock [J]. Applied Geophysics, 2005, 2 (4): 223-229.

[12] 谢然红. 低电阻率油气层测井解释方法研究 [J]. 测井技 术,2001,25(3):199-203.

[13] 秦东风. 低电阻率油层的综合识别方法研究[J]. 石油天然气 学报,2009,31(3):79-83.

[14] Archie G E. The Electrical Resistivity Log as an Aid in Determining Some Reservoir Characteristics[J]. Trans-actions AIME, 1942, 146: 54-61.

[15] 刘洪亮,王成荣,王鹏,等.吐哈盆地复杂孔隙结构储层测井评 价方法研究[J].测井技术,2015,39(3):313-317.

[16] 宋延杰, 唐晓敏. 低阻油层通用有效介质电阻率模型[J].中国 科学D辑: 地球科学. 2008,38(7):896-909.

[17] 罗少成,成志刚,周金昱,等.致密砂岩储层饱和度指数 $n$ 计算 方法研究 [J]. 西南石油大学学报 (自然科学 版）,2014,36(4):116-122.

[18] 刘柏林, 李治平, 匡松远, 等. 低含油饱和度油藏油水渗流特征: 以准噶尔盆地中部 1 区块为例 $[\mathrm{J}]$. 油气地质与采收 率,2007,14(1):69-72.

[19] 王勇,章成广,李进福,等.岩电参数影响因素研究 [J].石油天 然气学报,2006,28(4):75-77. 